\title{
A narrative review of gaps in the provision of integrated care for noncommunicable diseases in India
}

Manoj Kumar Pati ${ }^{1 *}$ D, N. Swaroop ${ }^{1}$, Arin Kar ${ }^{1}$, Preeti Aggarwal ${ }^{2}$, Krishnamurthy Jayanna ${ }^{1,3}$ and Wim Van Damme

\author{
* Correspondence: pati. \\ manojkumar@gmail.com \\ ${ }^{1}$ Karnataka Health Promotion Trust \\ IT Park, 5th floor, 1-4, Rajajinagar \\ Industrial Area behind KSSIDC \\ Admin Office, Rajajinagar, \\ Bangalore, Karnataka 560044, India \\ Full list of author information is \\ available at the end of the article
}

\begin{abstract}
Background: Low- and middle-income countries (LMICS) account for a higher burden of noncommunicable diseases (NCD) and home to a higher number of premature deaths (before age 70) from NCDs. NCDs have become an integral part of the global development agenda; hence, the scope of action on NCDs extends beyond just the health-related sustainable development goal (SDG 3). However, the organization and integration of NCD-related health services have faced several gaps in the LMIC regions such as India. Although the national NCD programme of India has been in operation for a decade, challenges remain in the integration of NCD services at primary care. In this paper, we have analysed existing gaps in the organization and integration of NCD services at primary care and suggested plausible solutions that exist.
\end{abstract}

Method: The identification of gaps is based out of a review of peer-reviewed articles, reports on national and global guidelines/protocols. The gaps are organized and narrated at four levels such as community, facility, health system, health policy and research, as per the WHO Innovative Care for Chronic Conditions framework (WHO ICCC).

Result: The review found that challenges in the identification of eligible beneficiaries, shortage and poor capacity of frontline health workers, poor functioning of community groups and poor community knowledge on NCD risk factors were key gaps at the community level. Challenges at facility level such as poor facility infrastructure, lack of provider knowledge on standards of NCD care and below par quality of care led to poor management of NCDs. At the health system level, we found, organization of care, programme management and monitoring systems were not geared up to address NCDs. Multi-sectoral collaboration and coordination were proposed at the policy level to tackle NCDs; however, gaps remained in implementation of such policies. Limited research on the effect of health promotion, prevention and, in particular, non-medical interventions on NCDs was found as a key gap at the research level.

(Continued on next page)

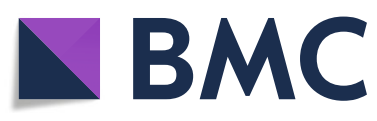

(c) The Author(s). 2020 Open Access This article is licensed under a Creative Commons Attribution 4.0 International License, which permits use, sharing, adaptation, distribution and reproduction in any medium or format, as long as you give appropriate credit to the original author(s) and the source, provide a link to the Creative Commons licence, and indicate if changes were made. The images or other third party material in this article are included in the article's Creative Commons licence, unless indicated otherwise in a credit line to the material. If material is not included in the article's Creative Commons licence and your intended use is not permitted by statutory regulation or exceeds the permitted use, you will need to obtain permission directly from the copyright holder. To view a copy of this licence, visit http://creativecommons.org/licenses/by/4.0/. The Creative Commons Public Domain Dedication waiver (http://creativecommons.org/publicdomain/zero/1.0/) applies to the data made available in this article, unless otherwise stated in a credit line to the data. 
(Continued from previous page)

Conclusion: This paper reinforces the need for an integrated comprehensive model of NCD care especially at primary health care level to address the growing burden of these diseases. This overarching review is quite relevant and useful in organizing NCD care in Indian and similar LMIC settings.

Keywords: Noncommunicable diseases, Health service delivery, Continuum of care, Urban health, Screening for NCDs, Follow-up and referral, Private health sector, Monitoring systems

\section{Background}

Four major noncommunicable diseases (NCDs) such as cardiovascular diseases, cancer, diabetes and chronic respiratory diseases are the leading global causes of death and are responsible for $70 \%$ of deaths worldwide in 2015 [1]. Three fourths of all these NCD deaths occurred in lower- and middle-income countries (LMICs) [2]. Recent reports on high-level meetings of United Nations General Assembly (UNGA) on NCDs [3, 4] suggest that an equitable and affordable primary care prevention, treatment and support system for NCDs is required to achieve universal health coverage (UHC). NCD targets cut across almost all sustainable development goals (SDG) [5]; hence, all high-burden countries should urgently prioritize actions towards this.

NCDs contribute to around 5.87 million deaths in India, accounting for $60 \%$ of all deaths in the country [1], and more than two-thirds of all NCD-related deaths in the entire South-East Asia Region (SEAR) [2]. The risk of an Indian dying between 30 and 70 years of age (premature deaths) from any one of the four major NCDs was about $23 \%$ in 2016 [6]. India is struggling to tackle the rising burden of NCDs due to epidemiological and demographic transitions. In such settings, the importance of primary health level care for NCDs cannot be overemphasized. In India, a majority (64\%) of healthcare payments is through out-of-pocket expenditure [7]. Evidence shows that the burden of NCDs and the prevalence of related risk factors are relatively higher in urban areas of India [8, 9]. The prevalence is on the rise among the poor, making them particularly vulnerable to catastrophic health expenditure, in addition to life-long morbidity and increased mortality [10]. Further, many NCDs goes unreported due to lack of clinical symptoms and are deeply related to individual lifestyle and habits. Hence, primary healthcare is the preferred choice to focus on health prevention and facilitate early screening. Additionally, evidence suggests primary care is the best avenue for delivering NCD care in the most integrated and comprehensive way [11-13].

The government of India launched the national NCD programme called the National Programme for Prevention and Control of Cancer, Diabetes, Cardiovascular Diseases \& Stroke (NPCDCS) in 2008. This national programme merged into the flagship National Health Mission (NHM) in 2013 aiming to integrate NCD-related programme activities related to health promotion, early diagnosis, treatment and referral and further facilitate partnerships with the private sector [14]. Despite such efforts and initiatives by the government, currently, there is no effective model which can demonstrate the integration of NCD-related services. It was apparent that the implementation approach followed in the national NCD programme was more suitable to the rural health system than an urban one until recently (2017). Very recently the programme recognized the 
importance of population-level screening and strengthening capacity-building efforts for primary care health care team and frontline health workers (FLWs) as crucial first steps to its aim of integration of services for NCD care [15]. Challenges remain in programme implementation plans; while the current government guidelines indicate sensitization of programme managers in service integration for effective management of NCDs [16], it lacks a proper plan of action. Similarly, there is a lack of comprehensive information pool and management information system to efficiently monitor the reach and implementation progress of the national NCD programme.

India was the first country to set national targets on NCDs in line with the global NCD action plan [17] and was among the first few to ratify and implement the WHO's Framework Convention on Tobacco Control, yet such a high burden of NCDs demonstrates the challenges in the implementation of national guidelines at the state and district level. Integrated service delivery models especially centred around primary care in urban areas are need of the hour to address the growing burden of NCDs [18]. Identifying gaps and solutions at different levels of intervention, integrating them into a package of interventions that is simple to implement and manage and building an efficient monitoring and evaluation system [19] are important for the development of sustainable NCD care models. Strengthening primary care for the integration of NCD services can be the beginning, and this paper highlights various gaps that hinder any such integration. Based on the gaps identified, we suggest an integrated NCD care model for primary care following an adapted version of the World Health Organization's Innovative Care for Chronic Conditions (ICCC) framework. The suggested model could be useful in organizing NCD care in India and similar settings.

\section{Methods}

\section{Review process}

Through a narrative review of the literature, we used the WHO ICCC framework as a theoretical framework of knowledge to identify and organize gaps under four major themes-at the community, facility, health system and gaps at the health policy and research. We adapted sub-themes and components of the ICCC framework to the local context (India and Karnataka).

\section{Hypothesis}

We hypothesize that gaps at community, facility, health system and policy level hampers effective integration and continuity of care for NCDs.

We have contextualized 'Integration of NCD Services' by following definitions [20, 21]:

1. Integrating NCD services into primary health care

2. Integrating NCD services from a health systems lens: Integration of all interventions at community level (mostly towards disease prevention and promotion, early identification and enrol to care), facility (towards disease management and follow-up) and overall systems level (towards supplies of medicine and logistics, programme management, monitoring systems) and finally at the level of policy and research. 
3. Integration of NCD services through a unified management approach: Integration of cross-cutting components like health promotion, prevention, screening of population, training, referral services, emergency medical services, public awareness programme management, monitoring and evaluation

Similarly, for the purpose of this study, we have defined 'Continuity in Care' more in terms of 'management continuity' and 'informational continuity' rather than 'relational continuity (the therapeutic relationship between a patient and one or more providers)' [22]. We have used an established 'continuum of care' framework [23] as an initial theoretical framework to focus on management continuity (i.e. starting from early screening, through confirmation and prompt treatment, patient follow-ups, to referral and complication management) and informational continuity (monitoring systems to track individualized treatment goals).

\section{Inclusion criteria}

We included all articles and reports published after the year 2000 in the review and used information from peer-reviewed articles, reports, guidelines and policy documents to identify the gaps.

\section{Search criteria and search items}

We conducted the literature review using MEDLINE (PubMed Central) and Google Scholar as search engines. Common search terms were 'chronic disease care', 'primary care', 'NCD in primary care', 'NCD and urban health', 'early screening of NCDs in the community', 'health system challenges in NCD care', 'programme management and NCD', 'NCD and policy' and 'India' as an adjunct search item. The initial literature review yielded some 343 articles (including reports) of which some 117 were initially screened for abstracts and results, and 77 full-length articles and reports were included in the final review. Finally, we reviewed 54 documents including full-length peerreviewed articles, reports and policy documents (Fig. 1).

\section{Results}

\section{Gaps at the level of community}

\section{Population enumeration to cover the eligible population}

Evidence shows that in India, geographical boundaries, especially those in urban areas, are often blurred. Revenue boundaries, judiciary boundaries and health services-related boundaries often do not match [24-26]. Additionally, a high level of in- and outmigration in urban areas pose a challenge to the enumeration of households $[27,28]$ making it difficult for resource allocation and organization of care. The planning of developmental programmes, including health service interventions, often suffers at the operational stage of defining the target population. Outreach activities including early screening and regular follow-up are impeded by the interplay of a variety of factors such as blurred boundary, capacity constraint of the community health workers and lack of continuous supportive supervision. 


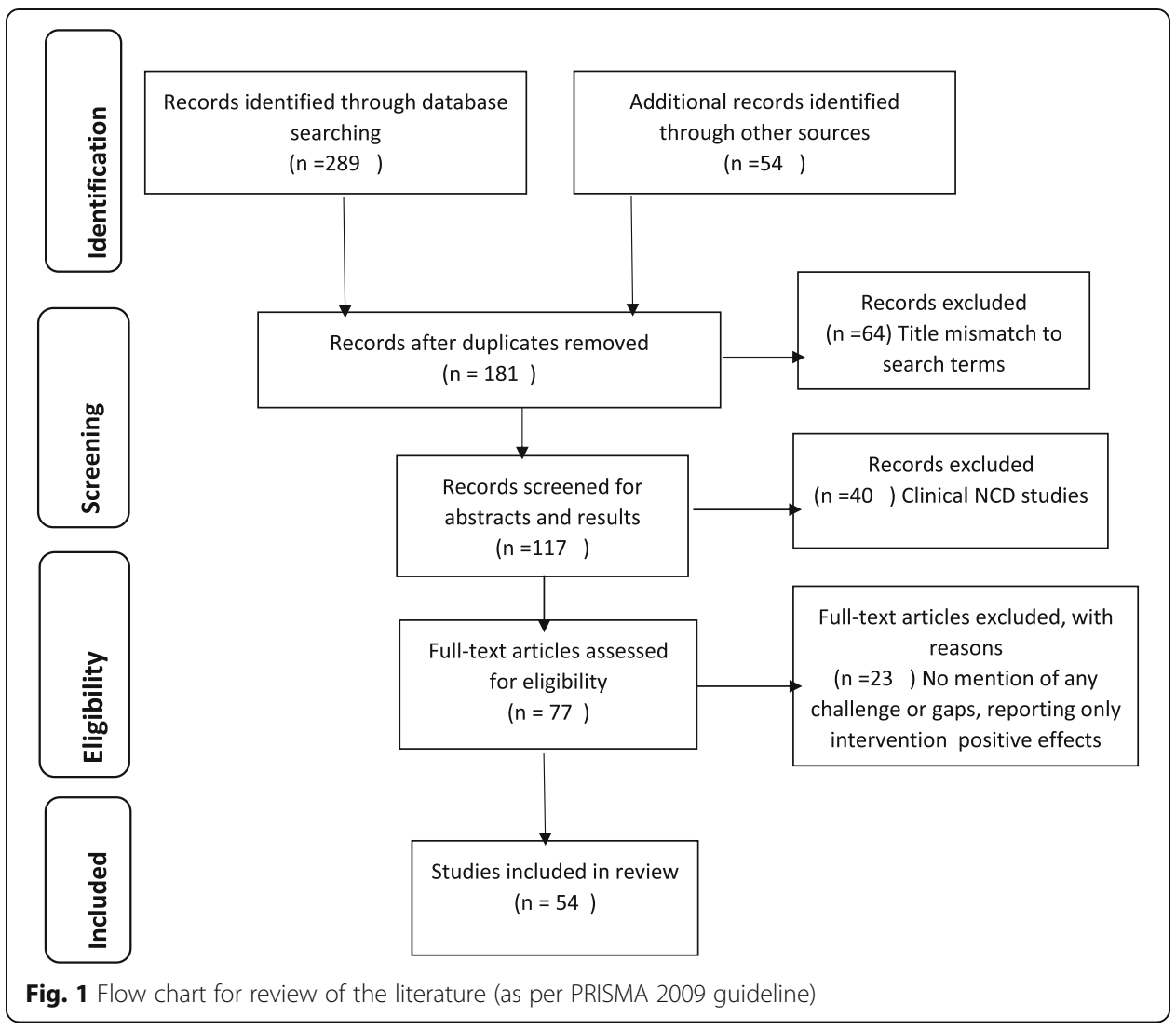

The knowledge gap in the community on risk factors

Although the awareness of diabetes is on the rise, especially in urban areas, the understanding of risks remains poor [29]. A study in India found that $25 \%$ of the population was unaware of diabetes and only $22.2 \%$ of the population and $41 \%$ of known diabetic subjects felt that diabetes could be prevented [30]. Even among the educated population comprising mainly postgraduates, medical professionals and lawyers, only $42.6 \%$ knew that diabetes could be preventable. The knowledge of risk factors of diabetes was even lower, with only $11.9 \%$ of study subjects reporting obesity and physical inactivity as the risk factors; 23\% knew that diabetes could lead to foot problems, and only 5.8\% knew it could cause a heart attack [30]. There is a lack of standard protocol on diabetes education and self-management in the community leading to noncompliant with health-seeking and intake of medicine [31]. In another study, among diabetics, $41.4 \%$ had not visited their health care provider in the last year; only $13 \%, 16.2 \%, 32.1 \%$ and $3.1 \%$ of respondents had undergone HbA1c test, eye examination, serum cholesterol test and foot examination, respectively [31]. Studies show that if risk factor targets were achieved, premature mortality from NCDs would decrease by $22 \%$ in men and $19 \%$ in women [32]; however, awareness does not necessarily always result in behavioural changes [33, 34]. However, few efforts have been made to address this knowledge gap on risk factors and improve health-seeking behaviour for NCDs in India $[35,36]$. This has resulted in major hurdles for effective management of NCDs, especially in vulnerable geographies. 
Gaps at the level of frontline health worker: shortage of manpower and lack of training

Another challenge to the early screening of NCDs in India is an inadequate number of trained FLWs to conduct population-level screening, health promotion and prevention activities as envisaged by the national NCD programme. As per government norm, each accredited social health activist (ASHA) is allocated per 1000 population in a rural area but in case of urban areas, this cadre is only allocated for 2500 in vulnerable and marginalized communities. In addition to this challenge, there is frequent vacancy of ASHA posts in urban areas given better availability of other job opportunities. Although recent government guidelines have delineated the role of FLWs such as auxiliary nurse midwives (ANMs) and ASHAs in early screening, referral and follow-up [14] of NCDs, FLWs across facilities in the country are yet to be fully sensitized on NCD care. For example, recent guidelines prescribe a tool for ASHAs, called the 'Community-Based Assessment Checklist' for early risk identification of common NCDs; however, the training is still ongoing and it is yet to be implemented in the field [15]. ASHAs lack handholding support in the community and are visibly overburdened by the number of tasks they are expected to perform; they are at a time expected to perform the role of an educator, link worker, service provider and activist which affect their performance [37-39]. ANMs are very recently trained on early screening of NCDs and care thereafter; however, the population screening programme is yet to be fully rolled out. Currently, the role of these frontline workers has been largely limited to carrying out maternal and child health interventions $[40,41]$.

\section{Limited role of community groups in NCD care}

Community groups such as Village Health Sanitation and Nutrition Committee (VHSNC) and MAS (Mahila Arogya Samiti ${ }^{1}$ ) are not well acquainted with the NCD programme and lack training to focus on the integration of NCDs into existing community monitoring mechanisms. Often MAS are non-functional in the urban areas making it difficult to sensitize them on NCD care.

In summary, community-level gaps pose a major challenge to early screening for NCDs and linking patients to care in time.

\section{Gaps at the level of a healthcare facility Gaps in the referral and follow-up of NCD cases}

Effective management of NCDs starts with effective referral system [20,21] which include referral of suspected cases for confirmatory tests, referral of patients to higher facilities for complication care and referral back to the community to maintain the continuity of care. Referral and follow-up of suspected cases to the nearest public (or private) facility for confirmation should follow post-screening of NCDs. At this stage, FLWs encounter several challenges. Lack of standardized referral protocols, referral forms and inadequate training of FLWs in the public health system are often the reasons

\footnotetext{
${ }^{1}$ MAS is one of the key interventions under National Health Mission aimed at promoting community participation in health at all levels, including planning, implementing and monitoring of health programmes. MAS is expected to take collective action on issues related to health, nutrition, water, sanitation and social determinants at the slum level. It is envisaged as being central to 'local collective action', which would gradually develop to the process of decentralized health planning. Each MAS covers around 50-100 households in slums or slum-like settlement, and function as a local women's collective with an elected chairperson or secretary while ASHA facilitates as member secretary.
} 
behind delay to the confirmation process. As a result, many patients either visit private facilities leading to out-of-pocket expense on confirmation tests or do not visit any facility leading to treatment delay. Those who do reach facilities are often not adequately oriented and counselled resulting in unnecessary anxiety about the diagnosis. These difficulties affect the continuity of care. NCD clinics are established at the secondary level or higher levels to facilitate care and treatment for referred cases as part of the national NCD programme [14]. However, the effectiveness of such clinics in delivering comprehensive care remains unknown. In summary, a weak referral and care system defeats the purpose of early screening.

\section{Gaps in facility readiness and provider capability}

Adequate facility readiness and provider preparedness are prerequisites for early initiation of treatment and high quality of NCD care. However, the public health system in India, which is supposed to be the principal custodian of people's health, is faced with multiple challenges at every level, mainly related to the readiness of facilities in terms of infrastructure and the preparedness of providers in terms of delivering standardized NCDs care [42-46]. Delayed diagnosis of diabetes and hypertension has been reported by many studies in India. In a Pan-India study, only $43.4 \%$ patients had their BP checked at the time of diagnosis; the figures were $17.6 \%, 5.6 \%$ and $4.2 \%$ for eye examination, kidney function tests and lipid tests, respectively, for diabetics with complications [47]. At the facility level, there is a lack of timely supply of drugs and medical equipment including diagnostic kits, lack of trained providers such as medical officers, pharmacists and laboratory technicians [47]. A study found that only $10-12 \%$ of people with diabetes received modern pharmacological treatment in India on an average. The availability of key oral anti-diabetic drugs such as Glibenclamide varied from $100 \%$ in the state of Karnataka to $3.8 \%$ in West Bengal. Although insulin therapy is accepted as one of the most effective and dependable treatment options, barriers to its use were identified [47]; In most patients, insulin was delayed until it was absolutely necessary or when the HbA1c levels had deteriorated further to approximately 9\%. Inadequate use of patient medical records and improper adherence to standard treatment protocols affect clinical decision-making; only six of the 15 health facilities in an urban health study had a system that tracked medical records of diabetes patients and providers in all these facilities expressed that there was no such thing called standard treatment protocols and treatment depends upon the individual analysis of patient condition [48]. This results in incomplete and irregular treatment leading to complications which ultimately bring economic and social liability along with suffering [49]. Although standard diagnosis and treatment protocols for NCDs are available, providers are yet to be trained on those protocols. Counselling and follow-up services also suffer in public health facilities due to lack of trained counsellors. For instance, absence of trained diabetic educators leaves the onus of counselling and educating patients on already overburdened physicians, and lack of standard counselling messages further result in inconsistent patient education and adherence to treatment [50]. Evidence shows that there is an age shift in the onset of NCDs in India, from an adult population group to relatively younger groups [51]. With the early onset, there is ample time for NCDs to develop complications given awareness is poor and the health system is not yet 
prepared to tackle the growing burden [52]. Systematic referral linkages have not yet been established across facilities for NCD treatment and complication management. Complications from NCDs lead to an increasing number of premature deaths [53] causing increased financial burdens on families and the nation as a whole. Citing delayed diagnosis, sub-optimal glycemic control and failure to screen for early-stage complications as three major reasons for these deaths, the study reported that $47 \%$ of the diabetes cases in the population were undiagnosed highlighting the poor awareness and detection of diabetes in India. It is important to note that $43 \%$ of deaths due to diabetes in India in 2016 were in people younger than 70 years again indicating possible inadequacies in diabetes management [53].

\section{Increased reliance on the private health sector for NCD care despite unknown quality of care}

Given the challenges associated with the public health system, the private health sector remains the preferred choice of care in India. Private practitioners, both qualified and unqualified, play a major role in health care delivery in India despite concerns about the practice of inconsistent treatment protocols, unnecessary diagnostic tests and high treatment costs [54]. The private sector now accounts for $60-80 \%$ of all out-patient health care in India [55] which leads to high health expenditures [48, 56-58]. This situation is similar for NCDs as many patients regularly access private health care of inconsistent and non-uniform quality.

In summary, facility-level gaps pose a challenge to the effective management of NCDs and related complications.

\section{Gaps in the public health system, programme management and monitoring systems}

India's public health system is not aligned and prepared to tackle chronic diseases posing a major hurdle to the organization of care and referral services for NCDs [59-61]. Current health systems are not able to plan for population-level NCD interventions so far due to the scale of the problem. More focus is being put on curative care and the setting up of NCD clinics, rather than development and implementation of an integrated model. Sub-optimal utilization of existing resources, below par programme management techniques and lack of planning, has affected the implementation of the welldesigned chronic care programme [62]. Further, the programme managers often lack capabilities in design, planning, implementation, monitoring and evaluation of NCD programmes due to inadequate training modules, process and tools, lack of data systems and underuse of technology [63].

\section{Gaps at policy and research level}

For effective delivery of NCD care at primary care level, attention and actions from policymakers on strategic planning [64] are required. Different types of NCDs are grouped within the current national programme, yet it may not make sense to approach them with a common strategy in the field; for example, the care approach to hypertension and to that of cancers should be very different from each other. This makes the delivery of primary care NCD services labour intensive and challenging. For example, the 
existing health system is not yet ready to handle an increasing burden of mental health illnesses in India as envisaged in the national NCD programme [65].

Further to this, the effective prevention, screening and treatment of NCDs require action and coordination from non-health stakeholders such as academia, legislation, urban planning, environment and food industry. Although indicated in policy documents such as the 5-year plan [66], progress in intersectoral coordination has remained limited. There are implementation gaps for interventions (promote physical activity in schools and society, restrict marketing of and access to food products high in salt, sugar or unhealthy fats) and legislations (clean indoor air legislation, tobacco advertising ban, raising tax on tobacco products) necessitating intersectoral coordination.

Another area that warrants attention is the need to expand investments in the area of implementation research around the prevention and promotion of NCDs. Currently, research and programme evaluations are skewed towards therapeutic/curative side. Research in understanding the role of alternative systems of medicine in health promotion and disease prevention is limited. Considering large vacancies of trained counsellors at urban primary care, the role of a counsellor in primary and secondary prevention of NCDs could be studied.

Based on the gaps identified in this article, we developed an integrated NCD care model for primary care based on an adapted version of the WHO ICCC framework (see Fig. 2). The WHO framework informs health systems to update its health care to meet the needs of chronic conditions. The building blocks of this framework are relevant for both prevention and disease management in health care settings. We are currently implementing this integrated NCD care model as a pilot intervention in one of the urban primary health centre catchment area in Mysore city of Karnataka state in India [18].

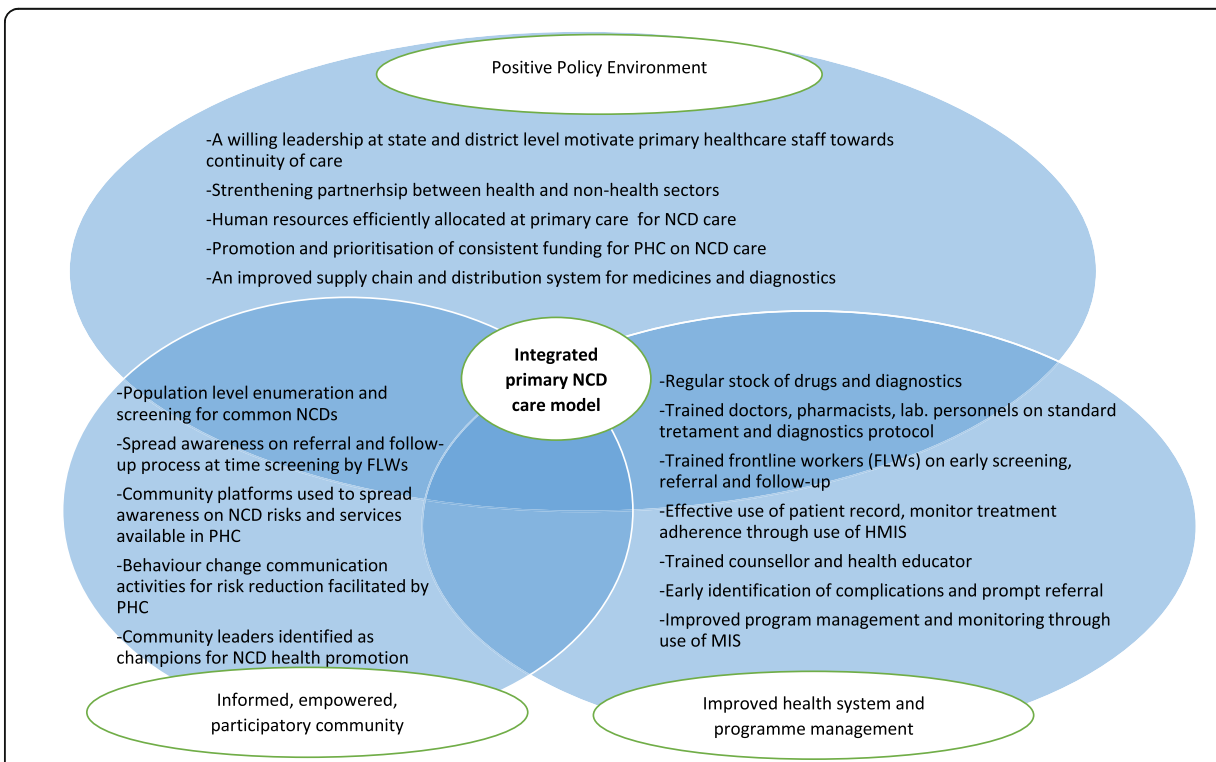

Fig. 2 Integrated primary NCD care model adapted from the WHO Innovative Care for Chronic Conditions (ICCC) Framework 


\section{Conclusion}

This review identified gaps in NCD care in India at various levels such as identifying target population, early screening through disease management to programme management and policy level. Given the large and versatile geography of the Indian subcontinent, NCD burden and response widely varies across states; while the burden is relatively higher in comparatively richer southern and western states, response and infrastructure in these states are also relatively better than northern and eastern counterparts. Similarly, rural and urban differences are huge in term of NCD prevalence; urban dynamics are different with large scale migration, the presence of a pluralistic health system and complex patient pathways. However, barring few exceptions, we did not find any major differences across states or urban-rural differences for the majority of gaps identified. We have analysed the presence and distribution of such gaps across the state and rural-urban regions through an additional excel file (Additional file 1); we found that majority of gaps holds good for most of the Indian states and across rural and urban geographies. There were few exceptions though such as enumerating the target population was found to be a major gap in urban areas compared to rural due to poorly defined geographical boundaries and continuous large scale migration. Poor community awareness on NCD risks and prevention found to be more in poor, remote and vulnerable geographies across states and urban-rural settlements. Overall patient awareness on disease management and self-care for NCDs found to be poor in both rural and urban geographies. While, northern and northeastern states found to be lagging in training frontline cadres on NCDs, in southern states, availability per se of such cadres was a challenge, especially in urban areas. Facility-level disease management gaps existed more or less similar across states and urban-rural areas. Although, health system response and policy dialogues for NCDs were in a little progressive stage in southern states; we did not find any major differences in action towards NCDs among different states. While the public facilities are not geared up for addressing the growing burden of NCDs, private health care for NCDs comes with very high cost and of questionable standards.

Overall, there is a lack of prioritization of NCD services at the primary healthcare level in India, such that people with NCDs in India are highly reliant on the private health sector where the quality and standard of care is uncertain and where the government has no control on overpricing. There is a lack of knowledge among people about available NCD services in the public health system and little attempt is made to design interventions to target high-risk and vulnerable communities. Frontline health workers lack training in standards of referral and follow-up for presumptive, newly diagnosed and existing cases of NCDs. Although standard referral and follow-up protocols are available, these are far from being effectively institutionalized and implemented in its entirety. Gaps in programme management lie in identifying the most cost-effective local interventions for NCD care and in setting up monitoring and evaluation systems for NCD programme at primary care level. Although training manuals are available for programme managers, programme managers across India are yet to be trained in designing and implementing comprehensive NCD care programmes.

It is thus very important for the NCD programme to expand from the current 'screening focused' approach at primary healthcare level to an integrated approach of 'community engagement' for health promotion, risk reduction and 'provider and facility 
readiness' for effective treatment, management and rehabilitation. Learnings and strategies from existing chronic disease care programme could be adapted such as the innovative Detect-Treat-Prevent-Build strategy being used in the Revised National Tuberculosis Control Programme (RNTCP) [67]. Preventing through customized behaviour change communication for common risk factors and individual patient counselling for adhering to treatment and follow-up, detecting through systematic screening of target groups (age group, tobacco use, obesity etc.) and involvement of private providers in detecting NCD cases could be adapted. Similarly, treating through a trained multi-disciplinary team and use of fixed dose combination of anti-diabetic or antihypertensive drugs [68] just like TB drugs could be practised at primary care settings. Finally, building a support system is of much importance; continued supply of medicines, adequate staffing, continuous capacity building of primary care team on NCD care competencies, supportive supervision and mentoring and a strong data monitoring system could all be adapted from the TB programme. However, instead of a vertical approach as with RNTCP, the DTPB strategies could be adapted for NCDs within the existing PHC structure.

Thus, integrating NCDs at primary health care helps in managing NCDs at an early stage and therefore is a better investment than diagnosing and managing them at a later stage [20]. Enumeration and tracking of eligible populations need to be strengthened [69] to aid the initiation of early screening and provision of effective care [70]. There is an urgent need to build the capacity of existing health workers on early identification of disease and risk factors using standardized tools. The effective screening will help in detecting the early onset of NCDs as well as tracing the high-risk groups in the community $[18,71]$. It is also critical to engage with the community and encourage their active participation in the NCD programme; behaviour change communication (BCC) activities focusing on NCD risks and prevention strategies [72] are vital. Systematic assessments of facility readiness and provider capability for the provision of NCD services are urgently needed. Assessments can be adapted from other programmes like $\mathrm{MNCH}$ programme [73], and standardized checklists and treatment protocols can be used. Guidelines can be framed to leverage the potential of key private healthcare facilities in streamlining referral and follow-up to ensure the provision of standardized care at an affordable price.

Similarly, programme monitoring and evaluation are important for strategic planning; it is timely to revisit monitoring indicators of the national NCD programme and strive for a sustainable surveillance system [19]. Understanding the burden of disease through population-level data systems is essential; evidence suggests that development of monitoring indicators and an information system are vital for effective programmatic decision-making under the NCD programme in India [24, 38]. Lessons and good practices can be adapted from other programmes such as the National AIDS control programme, where a national web-enabled strategic management information systems improved programme management and monitoring situation for AIDS control in India [74].

An understanding of the patient landscape and specific global models can inform technological policy innovations which can lower barriers to effective NCD screening, diagnosis and management [75]. The WHO Package of Essential NCD Interventions (WHO PEN) is such an action-oriented set of cost-effective integrated interventions at 
the primary health care level and is documented to be the most effective in tackling health system issues [20]. Integration could be happened across different national health programme as well to optimize health system resources, e.g. breast cancer and cervical cancer, which have gender implications, could be better screened and handled if integrated into $\mathrm{RMNCH}+\mathrm{A}$ programme [76]. A policy can be chalked out to encourage women from participating in community breast and cancer screening programme [77]. India should acknowledge the growing burden of mental health disorders and could set an example by [78, 79] including mental health in its national NCD programme. Policies to create a positive environment for promoting healthy lifestyles need to be prioritized. Also, the government should consider the impact on health while planning and implementing other non-health-related policies. Harnessing interventions from AYUSH, such as yoga, breathing exercises, meditation and others, and evaluating them through rigorous scientific methods to understand their efficacy in NCD prevention and health promotion is important.

\section{Supplementary information}

Supplementary information accompanies this paper at https://doi.org/10.1186/s40985-020-00128-3.

Additional file 1:. Mapping of reviewed studies across identified gaps. All reviewed studies presented in the result section are mapped for study locations (states and urban-rural location) across identified gaps

\section{Abbreviations}

ANM: Auxiliary nurse midwife; ASHA: Accredited social health activist; DTPB: Detection, Treatment, Prevention, and Build; LMIC: Low- and middle-income countries; MAS: Mahila Arogya Samiti; NCDs: Noncommunicable diseases; PHC: Primary health centre; RMNCH+A: Reproductive, Maternal, Newborn Child and Adolescent Health Programme; RNTCP: Revised National Tuberculosis Control Program; SDG: Sustainable Development Goals; SEAR: South-East Asia Region; UNGA: United Nations General Assembly

\section{Acknowledgements}

We would like to thank Mr. Mohan HL, Mr. Hemanth Madegowda and Miss Shivla Saksena for their useful feedback during the review process.

Research involving human participants and/or animals

Not applicable

\section{Authors' contributions}

MKP originally conceived the idea of this narrative review of gaps in NCD care in India and conducted a major part of the review. MKP and SN worked in identifying and collating the articles for review. KJ and SN helped MKP in reviewing and synthesizing selected articles for review. AK and PA provided useful and major inputs that change the course of this manuscript. WD looked through the overall findings and provided final inputs. All authors read and approved the final manuscript.

\section{Authors' information}

The first author is currently working as a senior public health researcher at the Karnataka Health Promotion Trust, Bangalore, India, with more than 8 years of experience in implementation and operational research in NCD care in southern India. The first author is currently pursuing his doctoral study on evaluating the organization of NCD care in urban primary health care set-ups in one of the southern Indian city. The last but one author is the local supervisor and the last author is one of the academic supervisors of the first author. Both supervisors have thorough expertise in health systems and policy research, universal health care, NCDs and primary health care. On the context of the doctoral study, this overarching review was done to serve as a background during the situational assessment phase.

Funding

No funding is available for this review.

\section{Availability of data and materials}

Not applicable

Ethics approval and consent to participate

Not applicable 


\section{Competing interests}

The authors declare that they have no competing interests.

\section{Author details}

${ }^{1}$ Karnataka Health Promotion Trust, IT Park, 5th floor, 1-4, Rajajinagar Industrial Area behind KSSIDC Admin Office, Rajajinagar, Bangalore, Karnataka 560044, India. ${ }^{2}$ Social Initiatives, Landmark Group, Bangalore, India. ${ }^{3}$ Centre for Global Public Health, University of Manitoba, Winnipeg, Canada. ${ }^{4}$ Health Policy Department, Institute of Tropical Medicine, Antwerp, Belgium.

Received: 16 October 2019 Accepted: 27 April 2020

Published online: 13 May 2020

\section{References}

1. World Health Organization. Noncommunicable diseases progress monitor 2015. World Heal Organ. 2015;232.

2. Mendis S. Global status report on noncommunicable diseases 2014 [Internet]. World Health Organization. 2014. Available from: https://apps.who.int/iris/bitstream/handle/10665/148114/9789241564854_eng.pdf;jsessionid=41ED67234 9C8B9DB73FF152DF16FA819? sequence=1.

3. World Health Organization. How WHO will report in 2017 to the United Nations General Assembly on the progress achieved in the implementation of commitments included in the 2011 UN Political Declaration and 2014 UN Outcome Document on NCDs. Vol. 136. 2017.

4. United Nation. Outcome document of high level meeting of General Assembly on NCD. 2014.

5. NCD Alliance. NCDs ACR SS THE SDGs. 2016;(2010):148114. Available from: https://ncdalliance.org/sites/default/files/ resource_files/NCDs_Across_SDGs_English_May2017.pdf.

6. World Health Organization. Noncommunicable diseases country profiles 2018 [Internet]. World Health Organisation. Geneva; 2018. Available from: https://www.who.int/nmh/countries/2018/ind_en.pdf?ua = 1 .

7. World Health Organisation. Out-of-pocket expenditure (\% of current health expenditure) [Internet]. World Health Organisation. 2019. Available from: https://data.worldbank.org/indicator/SH.XPD.OOPC.CH.ZS?locations=IN.

8. Mohan V, Kaur T, Anjana RM, Pradeepa RG. ICMR-INDIAB study (Phase I). 2016;1-59. Available from: https://www.icmr.nic in/sites/default/files/reports/ICMR_INDIAB_PHASE_I_FINAL_REPORT.pdf.

9. Kroll M, Kraas F. Non-communicable diseases in urban India: challenges for public health - UGEC viewpoints. July 19 [Internet]. 2016;2012-7. Available from: https://ugecviewpoints.wordpress.com/2016/07/19/non-communicable-diseasesin-urban-india-challenges-for-public-health/.

10. Bhojani U, Beerenahalli TS, Devadasan R, Munegowda CM, Devadasan N. No longer diseases of the wealthy: prevalence and health-seeking for self-reported chronic conditions among urban poor in Southern India. BMC Health Serv Res [Internet]. 2013;13(1):1. Available from: BMC Health Services Research.

11. Kane J, Landes M, Carroll C, Nolen A, Sodhi S. A systematic review of primary care models for non-communicable disease interventions in Sub-Saharan Africa. BMC Fam Pract. 2017;18(1):1-12.

12. Nandan D, Adhish VS, Dhar N. Relevance of primary health care in controlling noncommunicable diseases in India. Indian J Community Med [Internet]. 2011 Dec [cited 2017 Oct 26];36(Suppl 1):S4-6. Available from: http://www.ncbi.nIm. nih.gov/pubmed/22628910.

13. Maher D, Sekajugo J, Harries AD, Grosskurth H. Research needs for an improved primary care response to chronic noncommunicable diseases in Africa. Trop Med Int Heal [Internet]. 2010;15(2):176-81 Available from: http://onlinelibrary. wiley.com/store/10.1111/j.1365-3156.2009.02438.x/asset/j.1365-3156.2009.02438.x.pdf?v=1\&t=iid2hyhj\&s=f8fff1 db86 89c70bc330f880feaa7a8b3809c619.

14. Minsitry of Health and Family Welfare G of I. NPCDCS operational revised guidelines 2013-2017. 2017;130.

15. National Centre for Disease Control, MOHFW, GOl. National Programme for Prevention and Control of Cancer, Diabetes, Cardiovascular Diseases and Stroke training module for medical officers for prevention, control and population level screening of hypertension, diabetes and common cancer (Oral, Breast \&am. 2017 [cited 2017 Dec 6]; Available from: http://www.nicd.nic.in/writereaddata/mainlinkFile/File637.pdf.

16. NHM, MOHFW, GOI. Operational guidelines for prevention, screening and control of common non-communicable diseases. 2015 [cited 2017 Dec 5]; Available from: http://www.nicpr.res.in/images/pdf/guidelines_for_population_level_ screening_of_common_NCDs.pdf.

17. WHO. WHO | India: first to adapt the Global Monitoring Framework on noncommunicable diseases (NCDs) [Internet]. WHO. 2015. Available from: https://www.who.int/features/2015/ncd-india/en/.

18. Jayanna K, Swaroop N, Kar A, Ramanaik S, Pati MK, Pujar A, et al. Designing a comprehensive Non-Communicable Diseases (NCD) programme for hypertension and diabetes at primary health care level: evidence and experience from urban Karnataka, South India. BMC Public Health. 2019;19(1):1-12.

19. Krishnan A, Gupta V, Ritvik, Nongkynrih B, Thakur J. How to effectively monitor and evaluate NCD programmes in India. Indian J Community Med [Internet]. 2011 Dec [cited 2017 Dec 5];36(Suppl 1):S57-62. Available from: http://www.ncbi. nlm.nih.gov/pubmed/22628913.

20. Narain JP. Integrating services for noncommunicable diseases prevention and control: use of primary health care approach. Indian J Community Med [Internet]. 2011 Dec [cited 2017 Dec 5];36(Suppl 1):S67-71. Available from: http:// www.ncbi.nlm.nih.gov/pubmed/22628915.

21. Srivastava RK, Bachani D. Burden of NCDs, policies and programme for prevention and control of NCDs in India. 2011; $36: 1-7$.

22. Haggerty JL, Reid RJ, Freeman GK, Starfield BH, Adair CE, McKendry R. Continuity of care: a multidisciplinary review. Br Med J [Internet]. 2003;327(7425):1219-21. Available from: https://www.bmj.com/content/327/7425/1219.

23. Gabert R, Ng M, Sogarwal R, Bryant M, Deepu R V., McNellan CR, et al. Identifying gaps in the continuum of care for hypertension and diabetes in two Indian communities. BMC Health Serv Res [Internet]. 2017;17(1):1-11. Available from: https://www.ncbi.nlm.nih.gov/pubmed/29282052. 
24. World Bank. Urbanization beyond municipal boundaries : nurturing metropolitan economies and connecting peri-urban areas in India. [Internet]. World Bank; 2013 [cited 2017 Dec 6]. 106 p. Available from: https://books.google.co.in/ books?id=-lvpT7Zlh-oC\&pg=PA26\&lpg=PA26\&dq=urban+boundaries+are+poorly+defined+in+India\&source=bl\&ots= BAINEhuP7r\&sig=Zmoq4IKYcQWvWAwEld8qGufEZOY\&hl=en\&sa=X\&ved=0ahUKEwju-uHDwvPXAhULNY8KHTAiBK0Q6 AEIQZAF\# $=$ =nepage\&q=urban.boundaries are poorly defined in India\&f=false.

25. Sreevatsan A. How much of India is actually urban? - Livemint [Internet]. 2017 [cited 2017 Dec 6]. Available from: http:// www.livemint.com/Politics/4UjtdRPRikhpo8vAEOV4hK/How-much-of-India-is-actually-urban.html.

26. Hiranandani K, Tandel V. Chasing definitions in India - Livemint [Internet]. 2015 [cited 2017 Dec 6]. Available from: http://www.livemint.com/Opinion/1XflHBl9eRfFIlh117633l/Chasing-definitions-in-India.html.

27. Census of India. Migration. 2011 [cited 2017 Dec 6]; Available from: http://censusindia.gov.in/Ad_Campaign/drop_in_ articles/08-Migration.pdf.

28. Das KC, Saha S. Inter-state migration and regional disparities in India. Int Union Sci Study Popul [Internet]. 2013 [cited 2017 Dec 6]; Available from: https://www.iussp.org/sites/default/files/event_call_for_papers/Inter-state.migration_ IUSSP13.pdf.

29. Kaveeshwar SA, Cornwall J. The current state of diabetes mellitus in India. Australas Med J [Internet]. 2014 [cited 2017 Dec 5];7(1):45-8. Available from: https://www.ncbi.nlm.nih.gov/pmc/articles/PMC3920109/pdf/AMJ-07-40.pdf.

30. Mohan D, Raj D, Shanthirani CS, Datta M, Unwin NC, Kapur A, et al. Awareness and knowledge of diabetes in Chennai The Chennai Urban Rural Epidemiology Study [CURES - 9]. J Assoc Physicians India. 2005;:

31. Venkataraman K, Kannan AT, Mohan V. Challenges in diabetes management with particular reference to India. Int J Diabetes Dev Ctries. 2009;

32. Kontis V, Mathers CD, Rehm J, Stevens GA, Shield KD, Bonita R, et al. Contribution of six risk factors to achieving the 25×25 non-communicable disease mortality reduction target: A modelling study. Lancet. 2014.

33. Science for Environment Policy. Environmental awareness does not lead to smaller carbon footprints [Internet]. 2012 [cited 2017 Dec 6]. Available from: http://ec.europa.eu/environment/integration/research/newsalert/pdf/292 na6_en.pdf.

34. Sachans D, Optekamp C. Raising awareness, changing behavior? 2016 [cited 2017 Dec 6]; Available from: https://www. wodc.nl/binaries/Cahier.2016-11_2683_Volledige tekst_tcm28-239610.pdf.

35. Kanungo S, Bhowmik K, Mahapatra T, Mahapatra S, Bhadra UK, Sarkar K. Perceived morbidity, healthcare-seeking behavior and their determinants in a poor-resource setting: Observation from India. PLoS One. 2015;10(5):1-21.

36. Nath, Garg S, Deb S, Ray A, Kaur R. A study of the profile of behavioral risk factors of non communicable diseases in an urban setting using the WHO steps 1 approach. Ann Trop Med Public Heal. 2009;

37. Saprii L, Richards E, Kokho P, Theobald S. Community health workers in rural India: analysing the opportunities and challenges Accredited Social Health Activists (ASHAs) face in realising their multiple roles. Hum Resour Health [Internet]. 2015 Dec 9 [cited 2017 Dec 6];13(1):95. Available from: http://human-resources-health.biomedcentral.com/articles/10.11 86/s12960-015-0094-3.

38. Sarin E. Challenges facing the ASHA female community health workers, a vital part of the health system in India | USAID ASSIST Project [Internet]. USAID ASSIST Project. 2015 [cited 2017 Dec 6]. Available from: https://www.usaidassist.org/ blog/challenges-facing-asha-female-community-health-workers-vital-part-health-system-india.

39. Chingale AL. Job Satisfaction of Accredited Social Health Activists (ASHA's) Under NRHM. In: India International Public Health Conference. 2014.

40. Shet S, Sumit K, Phadnis S. A study on assessment of ASHA's work profile in the context of Udupi Taluk, Karnataka, India. 2017 [cited 2017 Dec 6]; Available from: http://www.ceghonline.com/article/S2213-3984(17)30056-8/pdf.

41. Shashank KJ, Angadi MM, Masali KA, Wajantri P, Bhat S, Jose AP. A study to evaluate working profile of accredited social health activist (ASHA) and to assess their knowledge about infant health care. Int I Curr Res Rev. 2013;5(12):97-103.

42. Peck R, Mghamba J, Vanobberghen F, Kavishe B, Rugarabamu V, Smeeth L, et al. Preparedness of Tanzanian health facilities for outpatient primary care of hypertension and diabetes: a cross-sectional survey. Lancet Glob Heal [Internet]. 2014 May [cited 2017 Dec 5];2(5):e285-92. Available from: http://www.ncbi.nlm.nih.gov/pubmed/24 818084 .

43. Bekele A, Getachew T, Amenu K, Defar A, Teklie H, Gelibo T, et al. Service availability and readiness for diabetes care at health facilities in Ethiopia. Ethiop J Heal Dev [Internet]. 2017 Jun 22 [cited 2017 Dec 5];31(2). Available from: http:// www.ejhd.org/index.php/ejhd/article/view/1223/930.

44. Katende D, Mutungi G, Baisley K, Biraro S, Ikoona E, Peck R, et al. Readiness of Ugandan health services for the management of outpatients with chronic diseases. 2015 [cited 2017 Dec 5]; Available from: https://pdfs.semanticscholar. org/24ac/4b44be68cc22cdc0a152fcba7f524ac145a5.pdf.

45. Bhojani U, Thriveni B, Devadasan R, Munegowda C, Amruthavalli, Devadasan N, et al. Challenges in organizing quality diabetes care for the urban poor: a local health system perspective. BMC Proc [Internet]. 2012 [cited 2017 Dec 5]; 6(Suppl 5):013. Available from: http://bmcproc.biomedcentral.com/articles/10.1186/1753-6561-6-S5-013.

46. Elias MA, Pati MK, Aivalli P, Srinath B, Munegowda C, Shroff ZC, et al. Preparedness for delivering noncommunicable disease services in primary care: access to medicines for diabetes and hypertension in a district in south India. BMJ Glob Heal [Internet]. 2018;2(Suppl 3):e000519. Available from: http://gh.bmj.com/lookup/doi/1 0.1136/bmjgh-2017-000519.

47. Viswanathan, Vijay, Rao VN. Problems associated with diabetes care in India. Diabetes Manag [Internet]. 2013 [cited 2017 Nov 20];3(1):31-40. Available from: http://www.openaccessjournals.com/articles/problems-associated-with-diabetes-carein-india.pdf.

48. Bhojani U, Devedasan N, Mishra A, De Henauw S, Kolsteren P, Criel B. Health system challenges in organizing quality diabetes care for urban poor in south india. PLoS One [Internet]. 2014 [cited 2017 Dec 5];9(9). Available from: https:// biblio.ugent.be/publication/5704394/file/5704396.

49. Upadhyay RP. An overview of the burden of non-communicable diseases in India. Iran J Public Health [Internet]. 2012; $41(3): 1-8$. Available from: http://www.ncbi.nlm.nih.gov/pmc/articles/PMC3481705/\%5Cn, http://www.ncbi.nlm.nih.gov/ pmc/articles/PMC3481705/pdf/ijph-41-1.pdf. 
50. Wangnoo SK, Maji D, Das AK, Rao P V, Moses A, Sethi B, et al. Barriers and solutions to diabetes management: an Indian perspective. Indian J Endocrinol Metab [Internet]. 2013 Jul [cited 2017 Dec 8];17(4):594-601. Available from: http://www. ncbi.nlm.nih.gov/pubmed/23961474

51. Mohan V, Sandeep S, Deepa R, Shah B, Varghese C. Epidemiology of type 2 diabetes: Indian scenario. 2007;(March):217-30.

52. Gaiha SM, Shukla R, Gilbert CE, Anchala R, Gudlavalleti MVS. Is India's policy framework geared for effective action on avoidable blindness from diabetes? Indian J Endocrinol Metab [Internet]. 2016 Apr [cited 2017 Dec 5];20(Suppl 1):S42-50. Available from: http://www.ncbi.nlm.nih.gov/pubmed/27144136.

53. Tandon N, Anjana RM, Mohan V, Kaur T, Afshin A, Ong K, et al. The increasing burden of diabetes and variations among the states of India: the Global Burden of Disease Study 1990-2016. Lancet Glob Heal [Internet]. 2018 Dec;6(12):e135262. Available from: https://inkinghub.elsevier.com/retrieve/pii/S2214109X18303875.

54. Sengupta A, Nundy S. The private health sector in India. BMJ [Internet]. 2005 Nov 19 [cited 2017 Dec 8];331(7526):11578. Available from: http://www.ncbi.nlm.nih.gov/pubmed/16293815.

55. Jain N, Kumar A, Nandraj S, Furtado KM. NSSO 71st round same data, multiple interpretations. 2015 [cited 2017 Dec 8]; Available from: http://niti.gov.in/writereaddata/files/document_publication/NSSO_71st_Round_Final.pdf.

56. Bronner Murrison L, Ananthakrishnan R, Sukumar S, Augustine S, Krishnan N, Pai M, et al. How do urban Indian private practitioners diagnose and treat tuberculosis? A cross-sectional study in Chennai. PLoS One [Internet]. 2016 [cited 2017 Dec 8];11(2):e0149862. Available from: http://www.ncbi.n/m.nih.gov/pubmed/26901165.

57. Kapur A, Shishoo S, Ahuja M, Sen V, Mankame K. Diabetes care in India: physicians perceptions, attitudes and practices. INT J DIAB DEV Ctries [Internet]. 1998 [cited 2017 Dec 8]; Available from: http://diabetes.org.in/journal/1998_oct-dec/article6.pdf.

58. Engel N, Ganesh G, Patil M, Yellappa V, Pant Pai N, Vadnais C, et al. Barriers to point-of-care testing in India: results from qualitative research across different settings, users and major diseases. Vermund SH, editor. PLoS One [Internet]. 2015 Aug 14 [cited 2017 Dec 8];10(8):e0135112. Available from: http://dx.plos.org/10.1371/journal.pone.0135112.

59. Anand K, Shah B, Yadav K, Singh R, Mathur P, Paul E, et al. Are the urban poor vulnerable to non-communicable diseases? A survey of risk factors for non-communicable diseases in urban slums of Faridabad. Natl Med J India [Internet]. 2007 [cited 2017 Dec 8];20(3):115-20. Available from: http://www.ncbi.nlm.nih.gov/pubmed/17867614.

60. Srinath Reddy K, Shah B, Varghese C, Ramadoss A. Responding to the threat of chronic diseases in India. Lancet [Internet]. 2005 Nov 12 [cited 2017 Dec 8];366(9498):1744-9. Available from: http://www.ncbi.nlm.nih.gov/pubmed/16291069.

61. Brashier B, Londhe J, Madas S, Vincent V, Salvi S. Prevalence of self-reported respiratory symptoms, asthma and chronic bronchitis in slum area of a rapidly developing Indian city. Open J Respir Dis [Internet]. 2012 Aug 10 [cited 2017 Dec 8]; 02(03):73-81. Available from: http://www.scirp.org/journal/doi.aspx?DOl=10.4236/ojrd.2012.23011.

62. Alwan A, MacLean DR, Riley LM, D'Espaignet ET, Mathers CD, Stevens GA, et al. Monitoring and surveillance of chronic non-communicable diseases: progress and capacity in high-burden countries. Lancet [Internet]. 2010; Available from: https://www.ncbi.nlm.nih.gov/pubmed/21074258.

63. Keynejad R, Semrau M, Toynbee M, Evans-Lacko S, Lund C, Gureje O, et al. Building the capacity of policy-makers and planners to strengthen mental health systems in low- and middle-income countries: A systematic review. BMC Health Serv Res [Internet]. 2016;16(1):1-11. Available from: https://doi.org/10.1186/s12913-016-1853-0.

64. Ebrahim S, Pearce N, Smeeth L, Casas JP, Jaffar S, Piot P. Tackling non-communicable diseases in low- and middleincome countries: is the evidence from high- income countries all we need? 2013;10(1):1-6. Available from: https:// www.ncbi.nlm.nih.gov/pmc/articles/PMC3558465/.

65. Patel V, Copeland J. The great push for mental health: why it matters for india. Indian Journal of Medical Research [Internet]. 2011;134(10):407-9. Available from: https://www.ncbi.nlm.nih.gov/pmc/articles/PMC3237235/.

66. Planning Commission Government of India. Twelfth five year plan (2012-2017): social sectors, volume III [Internet]. Vol. III, Twelfth five year plan (2012-2017). 2013. 1-292 p. Available from: http://planningcommission.gov.in/plans/planrel/12 thplan/pdf/12fyp_vol3.pdf.

67. Purty AJ. Detect-treat-prevent-build: strategy for TB elimination in India by 2025. Indian Journal of Community Medicine. 2018.

68. Varghese C, Nongkynrih B, Onakpoya I, McCall M, Barkley S, Collins TE. Better health and wellbeing for billion more people: integrating non-communicable diseases in primary care. BMJ [Internet]. 2019;364:1-3. Available from: https:// www.bmj.com/content/364/bmj.1327.

69. NHM, MOHFW. National Programme for Prevention and Control of Diabetes, cardiovascular disease and stroke- a manual for medical officer. Prev Control. 2009;1-52.

70. MOHFW, India G of. National Programme for Prevention and Control of Diabetes, cardiovascular disease and stroke a manual for medical officer developed under the Government of India - WHO Collaborative Programme. 2008 [cited 2017 Dec 5]; Available from: http://www.searo.who.int/india/topics/cardiovascular_diseases/NCD_Resources_ COMBINED_MANUAL_for_medical_officer.pdf.

71. Campbell B. Screening and monitoring of prediabetes. Am Assoc Clin Endocrinol. 2009;904:20-2.

72. Krishnan A, Ekowati R, Baridalyne N, Kusumawardani N, Suhardi, Kapoor SK, et al. Evaluation of community-based interventions for non-communicable diseases: experiences from India and Indonesia. Health Promot Int. 2011;26(3):276-89.

73. Jayanna K, Mony P, BM R, Thomas A, Gaikwad A, HL M, et al. Assessment of facility readiness and provider preparedness for dealing with postpartum haemorrhage and pre-eclampsia/eclampsia in public and private health facilities of northern Karnataka, India: a cross-sectional study. BMC Pregnancy Childbirth [Internet]. 2014;14(1):304. Available from: http://bmcpregnancychildbirth.biomedcentral.com/articles/10.1186/1471-2393-14-304.

74. Bachani D, Sogarwal R. National response to HIV/AIDS in India. Indian J Community Med [Internet]. 2010;35(4):469. Available from: http://www.ijcm.org.in/text.asp?2010/35/4/469/74341.

75. Brown E, Natoli N, McLaughlin R, Mehta K. Pathways and barriers to diabetes screening: observations from rural Kenya. Procedia Eng [Internet]. 2015;107:387-94. Available from: https://doi.org/10.1016/j.proeng.2015.06.096.

76. Binagwaho A, Ngabo F, Wagner CM, Mugeni C, Gatera M, Nutt CT, et al. Integration of comprehensive women's health programmes into health systems: cervical cancer prevention, care and control in Rwanda. Bull World Health Organ [Internet]. 2013;91(9):697-703. Available from: http://www.who.int/entity/bulletin/volumes/91/9/12-116087.pdf.

77. Bonfill X, Marzo M, Pladevall M, Martí J, Emparanza Jl. Strategies for increasing women participation in community breast cancer screening. Cochrane database Syst Rev [Internet]. 2001;(1):CD002943. Available from: http://doi.wiley. com/10.1002/14651858.CD002943\%5Cn, http://www.ncbi.nlm.nih.gov/pubmed/11279781. 
78. Ngo VK, Rubinstein A, Ganju V, Kanellis P, Loza N, Rabadan-Diehl C, et al. Grand challenges: integrating mental health care into the non-communicable disease agenda. PLoS Med. 2013;10(5):1-5.

79. Ivbijaro G. Mental health as an NCD (non-communicable disease): the need to act. Ment Health Fam Med. 2011;8(3):131-2.

\section{Publisher's Note}

Springer Nature remains neutral with regard to jurisdictional claims in published maps and institutional affiliations.

Ready to submit your research? Choose BMC and benefit from:

- fast, convenient online submission

- thorough peer review by experienced researchers in your field

- rapid publication on acceptance

- support for research data, including large and complex data types

- gold Open Access which fosters wider collaboration and increased citations

- maximum visibility for your research: over $100 \mathrm{M}$ website views per year

At $\mathrm{BMC}$, research is always in progress.

Learn more biomedcentral.com/submissions 